2. Sudzius G, Mieliauskaite D, Siaurys A, et al. Distribution of peripheral lymphocyte populations in primary Sjögren's syndrome patients. J Immunol Res 2015;2015:854706.

3. Björk A, Mofors J, Wahren-Herlenius M. Environmental factors in the pathogenesis of primary Sjögren's syndrome. J Intern Med 2020;287:475-92.

4. Mahnke YD, Brodie TM, Sallusto F, Roederer M, Lugli E. The who's who of T-cell differentiation: human memory T-cell subsets. Eur J Immunol 2013;43:2797-809.

5. Semenzato G, Zambello R, Starkebaum G, Oshimi K, Loughran TP Jr. The lymphoproliferative disease of granular lymphocytes: updated criteria for diagnosis. Blood 1997;89:256-60.

6. Swerdlow SH, Campo E, Harris NL, et al, eds. WHO classification of tumours of haematopoietic and lymphoid tissues. 4th ed. Lyon, France: IARC Press, 2008.

7. Molad Y, Okon E, Stark P, Prokocimer M. Sjögren's syndrome associated T cell large granular lymphocyte leukemia: a possible common etiopathogenesis. J Rheumatol 2001;28:2551-2.

8. Franco G, Palazzolo R, Liardo E, Tripodo C, Mancuso S. T cell large granular lymphocytic leukemia in association with Sjögren's syndrome. Acta Haematol 2010;124:5-8.

9. Baber A, Nocturne G, Krzysiek R, et al. Large granular lymphocyte expansions in primary Sjögren's syndrome: characteristics and outcomes. RMD Open 2019;5:e001044.

10. Lamy T, Moignet A, Loughran TP Jr. LGL leukemia: from pathogenesis to treatment. Blood 2017;129:1082-94.

11. Geginat J, Lanzavecchia A, Sallusto F. Proliferation and differ- entiation potential of human CD8+ memory T-cell subsets in response to antigen or homeostatic cytokines. Blood 2003;101: 4260-6.

12. Brenchley JM, Karandikar NJ, Betts MR, et al. Expression of CD57 defines replicative senescence and antigen-induced apoptotic death of CD8+ T cells. Blood 2003;101:2711-20.

13. Tasaki S, Suzuki K, Nishikawa A, et al. Multiomic disease signatures converge to cytotoxic CD8 T cells in primary Sjögren's syndrome. Ann Rheum Dis 2017;76:1458-66.

\section{Atypical presentation of hairy cell leukemia: a report and comprehensive review}

TO THE EDITOR: Pulmonary medicine consultation was sought for a 65-year-old man referred from an outside hospital with complaints of breathlessness, mediastinal lymphadenopathy, and intermittent low-grade fever accompanied by worsening anemia and low total leukocyte count. The patient had a history of one month of first-line anti-tuberculous therapy (ATT) based on a provisional diagnosis of pulmonary tuberculosis. His chest radiography and thoracic contrast-enhanced computerized tomography (CECT) scan



Fig. 1. Spectrum of circulating lymphoid cells in the peripheral blood in this case. Note the absence of characteristic circumferential hair-like projections in most cells (Leishman Giemsa stain, $\times 400$ ). 
revealed no pulmonary lesion but anterior mediastinal lymphadenopathy only. Physical examination revealed only pallor, without palpable lymphadenopathy or hepatosplenomegaly. A complete radiological examination performed outside did not reveal any abnormalities other than mediastinal lymphadenopathy, which was also corroborated by positron emission tomography (PET)-CT scan.

Routine laboratory investigation revealed normocytic normochromic anemia [hemoglobin $(\mathrm{Hb}), 60 \mathrm{~g} / \mathrm{L}$; mean corpuscular volume $(\mathrm{MCV}), 88 \mathrm{fL}$, leukopenia (leucocyte count, $\left.2 \times 10^{9} / \mathrm{L}\right)$ with relative lymphocytic prominence $(30 \%$ neutrophil, $60 \%$ lymphocytes, $4 \%$ eosinophils, $6 \%$ monocytes), and adequate platelet count $\left(350 \times 10^{9} / \mathrm{L}\right)$. Peripheral smears revealed scattered suspicious lymphoid cells constituting $10 \%$ of all lymphoid cells (Fig. 1). A review of bone marrow aspiration (BMA) slides performed at the referring hospital for evaluation of his cytopenia(s) revealed hemodilution with suppressed trilineage hematopoiesis and no abnormal cells, indicating a suspicion of marrow hypoplasia. Trephine core sections showed a variably cellular marrow with suppressed trilineage hematopoiesis with near-total replacement by diffuse sheets of a monotonous population of small to intermediate-sized atypical lymphoid cells with round to mildly irregular nuclear contours, dense chromatin, and inconspicuous nucleoli. In places, these cells exhibited perinuclear clear zones with a fried egg appearance.

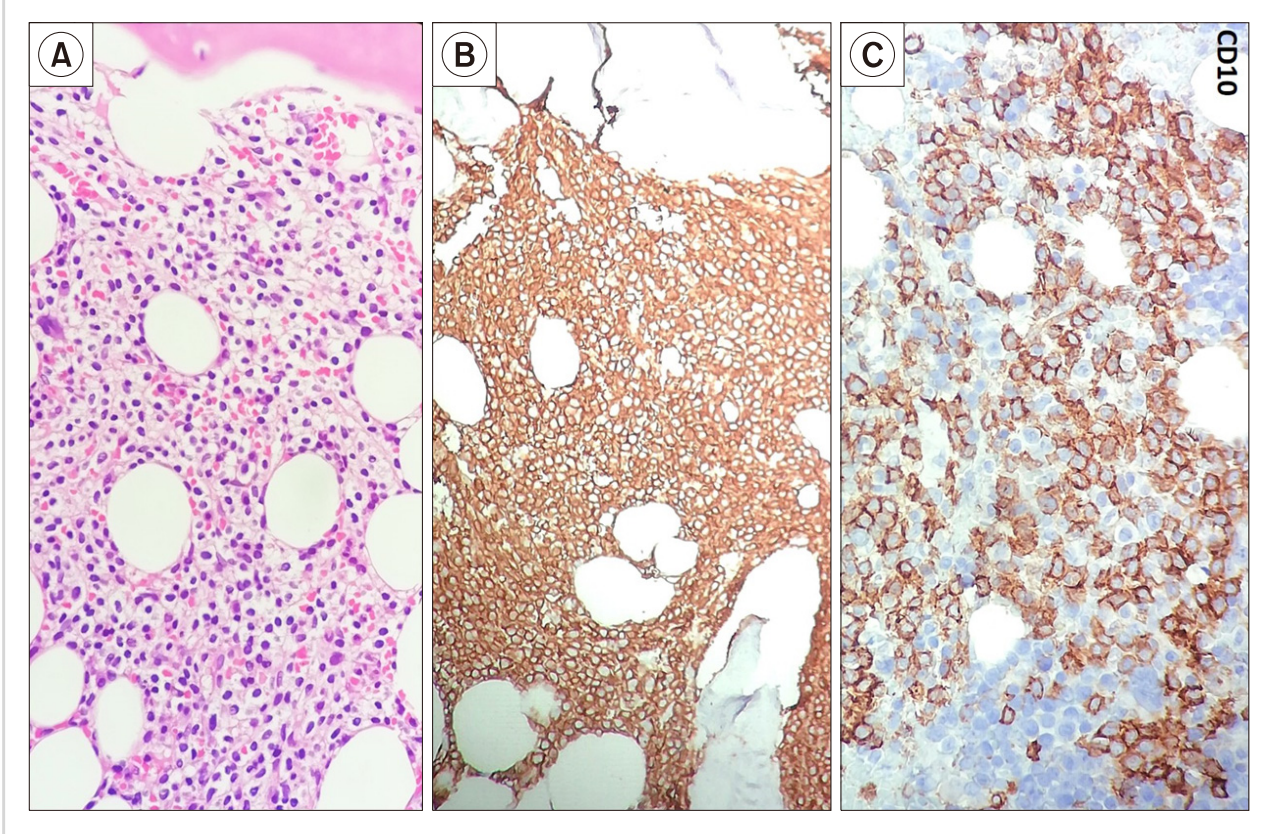

Fig. 2. Trephine marrow sections from the case demonstrating diffuse sheets of small mature-looking lymphoid cells with round to mildly irregular nuclear outlines and abundant clear cytoplasm with a 'fried egg' pattern classical of hairy cell leukemia (A, Hematoxylin and eosin, $\times 400$ ). On immunohistochemistry, these lymphoid cells show strong diffuse positivity for CD20 (B), CD11c (not shown), and CD10 (C, peroxidase-antiperoxidase, $\times 400$ ).

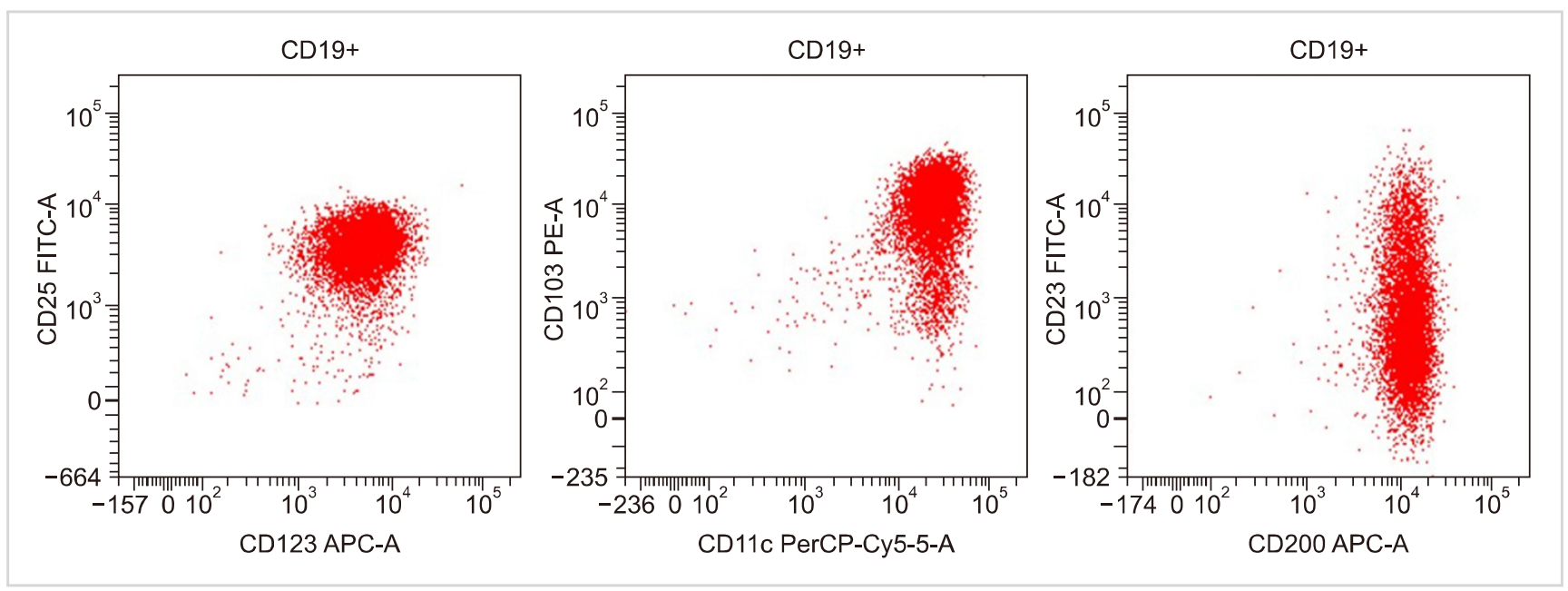

Fig. 3. Flow cytometric immunophenotyping plots with the population of interest (B cells) gated in red on CD19 versus side-scattered light (SSC). The B cells are positive for CD11c (bright), CD25 (bright), CD200, CD103, and CD123 diagnostic of hairy cell leukemia. Note the dim to moderate $\mathrm{CD} 23$ positivity in the CD19 ${ }^{+}$lymphoid cells as previously reported in the literature. 
Table 1. Atypical presentations of hairy cell leukemia: a comprehensive literature review.

\begin{tabular}{|c|c|c|c|c|c|c|c|c|}
\hline \multirow{2}{*}{ Ref. } & \multirow{2}{*}{$\begin{array}{l}\text { Age } \\
(y r) / \text { sex }\end{array}$} & \multirow{2}{*}{ Presentation } & \multicolumn{3}{|c|}{ Hairy cells } & \multirow{2}{*}{$\begin{array}{l}\text { Immunophenotyping } \\
\text { (FC/IHC) }\end{array}$} & \multirow{2}{*}{$\begin{array}{l}\text { Therapy; } \\
\text { outcome }\end{array}$} & \multirow{2}{*}{$\begin{array}{l}\text { Molecular } \\
\text { studies }\end{array}$} \\
\hline & & & PBS & $\mathrm{BMA}$ & BMBx/tissue $B x$ & & & \\
\hline$[3]$ & $52 / M$ & $\begin{array}{l}\text { Pancytopenia } \\
\text { (? aplastic); } \\
\text { no spleen }\end{array}$ & $-/+$ & $\begin{array}{c}\text { Scant, } \\
-/+\end{array}$ & $\begin{array}{l}\text { Hypoplastic atypical } \\
\text { lymphoid nodule }\end{array}$ & $\begin{array}{l}\text { CD200/CD11c/CD25/ } \\
\text { CD20/kappa: + }\end{array}$ & Cladribine; CR & $\mathrm{BRAF}+$ \\
\hline [3] & $56 / M$ & $\begin{array}{l}\text { Pancytopenia } \\
\text { (? aplastic) } \\
\text { no spleen }\end{array}$ & - & - & $\begin{array}{l}\text { Hypoplastic focal } \\
\text { fried egg }\end{array}$ & CD20/annexin A1; + & Cladribine; CR & Not tested \\
\hline$[3]$ & $48 / M$ & $\begin{array}{l}\text { Lymphocytosis; } \\
\text { massive } \\
\text { spleen, } \\
\text { multiple LNs }\end{array}$ & $-/+$ & $-/+$ & LPN; fried egg & $\begin{array}{l}\text { CD200/CD11c/CD25/ } \\
\text { CD20/kappa; + }\end{array}$ & Supportive & Not tested \\
\hline$[3]$ & $55 / \mathrm{F}$ & $\begin{array}{l}\text { Pancytopenia } \\
\text { (? aplastic); } \\
\text { no spleen }\end{array}$ & - & ?/+ & $\begin{array}{l}\text { Hypoplastic, atypical } \\
\text { lymphoid nodule }\end{array}$ & $\begin{array}{l}\text { CD200/CD11c/CD25/ } \\
\text { CD20/kappa; + }\end{array}$ & Cladribine; CR & $\mathrm{BRAF}+$ \\
\hline$[4]$ & $43 / \mathrm{F}$ & $\begin{array}{l}\text { Arthalgia } \\
\text { pancytopenia, } \\
\text { no spleen }\end{array}$ & $-/+$ & $-/+$ & Fried egg & $\begin{array}{l}\text { CD200/CD11C/CD25/ } \\
\text { CD20/FMC7/HLA-DR; + }\end{array}$ & $\begin{array}{l}\text { Cladribine; } \\
\text { not described }\end{array}$ & Not tested \\
\hline$[5]$ & $68 / M$ & $\begin{array}{l}\text { Lesion in skull } \\
\text { base, bones, } \\
\text { pleura; low } \\
\text { platelet; no } \\
\text { spleen } \\
\text { mediastinal LN }\end{array}$ & - & $-/+$ & LPN (tissue and BM) & \multicolumn{2}{|c|}{$\begin{array}{cc}\text { Cyclin D1+, CD 19/CD 20; } & \text { Cladribine; } \\
\text { +, CD5/CD23/CD10; -, } & \text { rituximab; } \\
\text { CD 11c/25/103; + } & \text { CR }\end{array}$} & $\begin{array}{l}t(11 ; 14) ;- \\
\text { BRAF- }\end{array}$ \\
\hline$[6]$ & $55 / M$ & $\begin{array}{l}\text { Lytic to sclerotic } \\
\text { bone lesion; } \\
\text { rt femur, no } \\
\text { spleen }\end{array}$ & - & - & $\begin{array}{l}\text { LPN (bone biopsy) } \\
\text { fried egg } \\
\text { BM; no abnormality }\end{array}$ & $\begin{array}{l}\text { TRAP/annexin A1/CD20/ } \\
\text { CD11c; +, FC: no } \\
\text { abnormality }\end{array}$ & $\begin{array}{l}\mathrm{RT}+ \\
\text { Cladribine, } \\
\mathrm{CR}\end{array}$ & $\mathrm{BRAF}+$ \\
\hline$[8]$ & $44 / F$ & $\begin{array}{l}\text { Breast lesion; } \\
\text { no spleen }\end{array}$ & $-/+$ & Dry tap & $\begin{array}{l}<10 \% \text { atypical } \\
\text { lymphoid cells, MF } 2\end{array}$ & $\begin{array}{l}\text { Breast: CD20/PAX5/ } \\
\text { Cyclin D1 (variable+)/ } \\
\text { annexin A1/TRAP;+ } \\
\text { CD25; weak+ } \\
\text { BM-FC: + (kappa) }\end{array}$ & Not described & $\begin{array}{l}t(11 ; 14) ;- \\
\text { BRAF+ }\end{array}$ \\
\hline [9] & $69 / M$ & $\begin{array}{l}\text { Pancytopenia } \\
\text { (? aplastic) } \\
\text { no spleen }\end{array}$ & - & $-1+$ & $\begin{array}{l}\text { Hypoplastic, atypical } \\
\text { lymphoid nodule, } \\
\text { fried egg }\end{array}$ & \multicolumn{2}{|c|}{$\begin{array}{l}\text { IHC: DBA44/annexin A1; + Cladribine; CR } \\
\text { FC: hairy cell leukemia }\end{array}$} & Not tested \\
\hline [9] & $50 / M$ & $\begin{array}{l}\text { Weakness, } \\
\text { weight loss, } \\
\text { bicytopenia; } \\
\text { no spleen }\end{array}$ & $-/+$ & $-/+$ & LPN; fried egg & $\begin{array}{l}\text { TRAP }(\mathrm{PBS}) ;+ \\
\text { FC; hairy cell leukemia }\end{array}$ & Cladribine; CR & Not tested \\
\hline$[9]$ & $43 / M$ & $\begin{array}{l}\text { Recurrent URTI, } \\
\text { low platelet; } \\
\text { no spleen }\end{array}$ & $-/+$ & $\begin{array}{l}-/+ \\
\quad(\text { TRAP } \\
\quad+\text { ve })\end{array}$ & LPN; fried egg & $\begin{array}{l}\text { CD200/CD11c/CD25/ } \\
\text { CD20/kappa; + }\end{array}$ & Supportive & Not tested \\
\hline$[9]$ & $67 / M$ & $\begin{array}{l}\text { Pancytopenia } \\
\text { (? aplastic/MDS) } \\
\text { no spleen }\end{array}$ & $10 \%$ & $\begin{array}{l}-/+ \\
\quad(\text { TRAP } \\
+ \text { ve })\end{array}$ & $\begin{array}{l}\text { Hypoplastic; atypical } \\
\text { lymphoid nodule, } \\
\text { fried egg, MF } 1 \text { to } 2\end{array}$ & $\begin{array}{l}\text { PB-FC: CD 19/CD 11c/ } \\
\text { CD 25/CD 103; }+\end{array}$ & Cladribine; CR & Not tested \\
\hline$[10]$ & $54 / M$ & $\begin{array}{l}\text { LS epidural } \\
\text { mass with } \\
\text { radiculopathy, } \\
\text { multifocal } \\
\text { bone lesions; } \\
\text { no spleen }\end{array}$ & - & Dry tap & $\begin{array}{l}\text { LPN fried egg } \\
\quad(\text { tissue } \mathrm{Bx}+\mathrm{BM})\end{array}$ & $\begin{array}{l}\text { Bx: CD20/TRAP/CD25; + } \\
\text { BM-FC: CD19/CD11c/ } \\
\text { CD103/CD25; + }\end{array}$ & Cladribine; CR & Not tested \\
\hline $\begin{array}{l}\text { Present } \\
\text { case }\end{array}$ & $63 / M$ & $\begin{array}{l}\text { Breathlessness; } \\
\text { mediastinal } \\
\text { LN, } \\
\text { leukopenia; } \\
\text { no spleen }\end{array}$ & $-1+$ & $-/+$ & LPN; Fried egg & \multicolumn{2}{|c|}{$\begin{array}{l}\text { IHC; CD 20/CD 10/CD 11C; Cladribine, CR } \\
\quad \text { +, FC; CD200/ } \\
\text { CD } 19 / C D 11 c / C D 103 / \\
\text { CD 10/CD25; +CD 23; } \\
\text { dim to moderate+ }\end{array}$} & Not tested \\
\hline $\begin{array}{l}\text { ohine bic } \\
\text { ometry; } \\
\text { elodyspl }\end{array}$ & $\begin{array}{l}+, \text { pos } \\
; \text { BRAF, } \\
\text {, immu } \\
\text { C syndr } \\
\text { ory tract }\end{array}$ & $\begin{array}{l}\text { sitive; -, negative } \\
\text {, BRAF V600E mu } \\
\text { unohistochemist } \\
\text { rome; MF, myelo } \\
\text { t infection. }\end{array}$ & $\begin{array}{l}\text { ion } \\
\text { LN, } \\
\text { osis }\end{array}$ & $\begin{array}{l}\text { ional } t \\
\text { sis; } C D \\
\text { h node } \\
\text { e as pe }\end{array}$ & $\begin{array}{l}\text { ry few; ?/+, suspiciou } \\
\text {, bright positivity in flo } \\
\text { PN, low grade lympho } \\
\mathrm{HO} \text {; PB, peripheral blo }\end{array}$ & $\begin{array}{l}\text { Us; BMA, bone marrow aspir } \\
\text { w cytometry; CR, complete } \\
\text { proliferative neoplasm; LS, I } \\
\text { od; PBS, peripheral blood sm }\end{array}$ & $\begin{array}{l}\text { iration; } \mathrm{BMBx}, \mathrm{bc} \\
\text { response; } \mathrm{F} \text {, fem } \\
\text { lumbosacral; } \mathrm{M} \text {, } \\
\text { near; } \mathrm{RT} \text {, radiothe }\end{array}$ & $\begin{array}{l}\text { ne marro } \\
\text { le; FC, flo } \\
\text { nale; MD } \\
\text { rapy; URT }\end{array}$ \\
\hline
\end{tabular}


There was no evidence of increased mitosis, granuloma, necrosis, or any large cell transformation. Reticulin staining showed myelofibrosis (MF) grade $0-1$ condensation [World Health Organization (WHO) classification]. Immunohistochemical investigation revealed lymphoid cells with strong and diffuse positivity for $\mathrm{CD} 20, \mathrm{CD} 11 \mathrm{c}$, and $\mathrm{BCl2}$; weak immunopositivity for CD10 and kappa light chain; and negativity for CD3, CD5, CD23, BCl6, CD138, cyclin D1, CD30, ALK1, CD34, CD117, and lambda light chain, consistent with a low-grade B cell lymphoproliferative neoplasm (LPN) of hairy cell phenotype (Fig. 2). The results of serum immunofixation electrophoresis and Coomb test were within normal limits. A repeat BMA sample was subjected to multiparametric flow cytometric immunophenotyping, which showed bright CD200, CD25, CD11c, CD19, and CD20 expression, consistent with hairy cell leukemia (HCL). Dim to moderate CD23 expression was also noted in a subset of these gated lymphoid cells (Fig. 3). The patient was referred to a specialized tertiary care cancer institute for further management; he was started on cladribine treatment and achieved a complete response and is presently under follow-up. Molecular testing for BRAF was not done as per the clinician's decision.

HCL is a rare, clinically indolent, mature small B cell lymphoproliferative neoplasm (LPN) that accounts for $2 \%$ of all lymphoid leukemias. It is characterized by the proliferation of small lymphoid cells with abundant cytoplasm with circumferential fine hairy projections involving the peripheral blood, bone marrow, and expanding splenic red pulp. The hallmark features are marked splenomegaly, peripheral blood cytopenia(s), monocytopenia, and circulating $\mathrm{CD} 19^{+}$, bright $\mathrm{CD} 20^{+} / \mathrm{CD} 22^{+} / \mathrm{CD} 200^{+}, \mathrm{CD} 25^{+}, \mathrm{CD}_{103}{ }^{+}$, and $\mathrm{CD}_{11 \mathrm{c}^{+}}$hairy cells demonstrated classically by flowcytometric analysis. Annexin A1 is the most specific marker for HCL, which helps in differentiating HCL mimics such as variant HCL (vHCL) and splenic marginal zone lymphoma, which are both annexin A1-negative [1].

Atypical or unusual presentation in HCL (aHCL) is uncommonly reported in the literature (1990 to 2018), which may pose a significant diagnostic challenge in routine practice (Table 1) [2-10]. Splenohepatomegaly is conspicuously absent which, in the presence of peripheral blood cytopenia(s), resembled hypoplastic anemias. Significant lymphadenopathy; skeletal manifestation in the form of arthralgia, multifocal lytic to sclerotic bone lesions; neurological manifestations; isolated epidural, skull base, or breast lesions have dominated the clinical presentations in other reports [2, 4-9]. HCs were conspicuously absent to very sparse; both in peripheral blood smear (PBS) and scant bone marrow aspirate (BMA) smears requiring tartrate-resistant acid phosphatase (TRAP) cytochemistry. The trephine sections resembled hypoplastic marrow with atypical lymphoid cells forming discrete or nodular aggregates unlike those of classical HCL. Diffuse infiltration by low-grade LPN gave a typical 'fried egg' appearance in others. Others showed classic histomorphology in extramedullary sites without evidence of marrow involvement. Flowcytometric and/or IHC analysis demonstrated classical HCL phenotypes with bright CD200 coexpression in all cases reported in the literature $[11,12]$. Immunophenotypic aberrancies such as heterogenous CD10 and CD23 coexpression are reported in up to $15 \%$ cases; whereas $\mathrm{CD}^{+}$and $\mathrm{CD} 103 / \mathrm{CD} 25^{-}$were sporadically reported and were not associated with any specific laboratory abnormalities. Atypical presentations or immunophenotypic aberrancies did not alter the favorable response to purine analogs such as cladribine [7, 11].

In summary, unusual or atypical clinical presentation is not uncommon in HCL. Furthermore, rare immunophenotypic aberrancies in HCL should be considered when interpreting flow cytometric/immunohistochemical patterns in such cases to avoid misdiagnosis.

Sandeep Abhijit Pattnaik ${ }^{1}$, Somanath Padhi ${ }^{1}$, Gaurav Chhabra ${ }^{1}$, Manoj Kumar Panigrahi ${ }^{2}$, Prabodh Kumar Das ${ }^{3}$, Rajesh Kumar Bhola ${ }^{4}$, Shruti Mishra ${ }^{1}$ Departments of ${ }^{1}$ Pathology and Laboratory Medicine, ${ }^{2}$ Pulmonary Medicine, and ${ }^{3}$ Medical Oncology/Hematology, All India Institute of Medical Sciences, ${ }^{4}$ Department of Laboratory Hematology, IMS and Sum Hospital, S'O'A Deemed to be University, Bhubaneswar, India

Correspondence to: Somanath Padhi Department of Pathology and Laboratory Medicine, All India Institute of Medical Sciences, Bhubaneswar 751019,

India

E-mail: pathol_somanath@aiimsbhubaneswar.edu.in

Received on Apr. 6, 2020; Revised on Apr. 13, 2020; Accepted on Apr. 16, 2020 https://doi.org/10.5045/br.2020.2020069

\section{Authors' Disclosures of Potential Conflicts of Interest}

No potential conflicts of interest relevant to this article were reported.

\section{REFERENCES}

1. Foucar K, Falini B, Stein H. Hairy cell leukemia. In: Swerdlow $\mathrm{SH}$, Campo E, Harris NL, et al, eds. WHO classification of tumours of haematopoietic and lymphoid tissues. Revised 4th ed. Lyon, France: IARC Press, 2017.

2. Moustafa A, Shwaylia HM, Aldapt M, Yassin MA. Outcome of atypical presentations of hairy cell leukemia. Blood (ASH Annual Meeting Abstracts) 2019;134(Suppl):5267.

3. Rahman K, Kumari S, Singh MK, et al. Atypical presentation of hairy cell leukemia: significance of CD200 on flow cytometry. J Cancer Res Ther 2018;14:1130-4.

4. Alfaraj M, Alsaeed H. Hairy cell leukemia: a case report of atypical presentation without splenomegaly. Blood Res 2017;52:139-41.

5. Chai KL, Morris T, Bazargan A. A case report of hairy cell leukemia: an unusual presentation. Ann Clin Case Rep 2017;2:1412.

6. Yonal-Hindilerden I, Hindilerden F, Bulut-Dereli S, Yıldız E, Dogan IO, Nalcaci M. Hairy cell leukemia presenting with iso- 
lated skeletal involvement successfully treated by radiation therapy and cladribine: a case report and review of the literature. Case Rep Hematol 2015;2015:803921.

7. Gupta AK, Sachdeva MU, Ahluwalia J, et al. Haematological profile of 21 patients with hairy cell leukaemia in a tertiary care centre of north India. Indian J Med Res 2015;142:426-9.

8. Pilichowska M, Shariftabrizi A, Mukand-Cerro I, Miller K. Primary hairy cell leukemia/lymphoma of the breast: a case report and review of the literature. Case Rep Pathol 2014; 2014:497027.

9. Venkatesan S, Purohit A, Aggarwal M, et al. Unusual presentation of hairy cell leukemia: a case series of four clinically un- suspected cases. Indian J Hematol Blood Transfus 2014;30(Suppl 1):413-7.

10. Rosen DS, Smith S, Gurbuxani S, Yamini B. Extranodal hairy cell leukemia presenting in the lumbar spine. J Neurosurg Spine 2008;9:374-6.

11. Chen YH, Tallman MS, Goolsby C, Peterson L. Immunophenotypic variations in hairy cell leukemia. Am J Clin Pathol 2006; 125:251-9.

12. Sandes AF, de Lourdes Chauffaille M, Oliveira CR, et al. CD200 has an important role in the differential diagnosis of mature B-cell neoplasms by multiparameter flow cytometry. Cytometry B Clin Cytom 2014;86:98-105. 